\title{
Quantitative Surface Plasmon Interferometry via Upconversion Photoluminescence Mapping
}

\author{
Anxiang Yin, ${ }^{1,2}$ Hao Jing, ${ }^{1}$ Zhan Wu, ${ }^{3,4}$ Qiyuan He ${ }^{D},{ }^{1}$ Yiliu Wang, ${ }^{1}$ Zhaoyang Lin ${ }^{1},{ }^{1}$ \\ Yuan Liu $\left(10,{ }^{3}\right.$ Mengning Ding, ${ }^{3} \mathrm{Xu} X u,{ }^{1}$ Zhe Fei, ${ }^{5}$ Jianhui Jiang, ${ }^{4}$ Yu Huang, \\ and Xiangfeng Duan $\mathbb{1}^{1,6}$ \\ ${ }^{1}$ Department of Chemistry and Biochemistry, University of California, Los Angeles, California 90095, USA \\ ${ }^{2}$ School of Chemistry and Chemical Engineering, Beijing Institute of Technology, Beijing 100008, China \\ ${ }^{3}$ Department of Materials Science and Engineering, University of California, Los Angeles, California 90095, USA \\ ${ }^{4}$ State Key Laboratory for Chemo/Biosensing and Chemometrics, College of Chemistry and Chemical Engineering, Hunan University, \\ Changsha 410082, China \\ ${ }^{5}$ Department of Physics \& Astronomy, Iowa State University, Ames, Iowa 50011, USA \\ ${ }^{6}$ California NanoSystems Institute, University of California, Los Angeles, California 90095, USA
}

Correspondence should be addressed to Xiangfeng Duan; xduan@chem.ucla.edu

Received 3 March 2019; Accepted 17 August 2019; Published 15 September 2019

Copyright (c) 2019 Anxiang Yin et al. Exclusive Licensee Science and Technology Review Publishing House. Distributed under a Creative Commons Attribution License (CC BY 4.0).

\begin{abstract}
Direct far-field visualization and characterization of surface plasmon polaritons (SPPs) are of great importance for fundamental studies and technological applications. To probe the evanescently confined plasmon fields, one usually requires advanced nearfield techniques, which is typically not applicable for real-time, high-throughput detecting or mapping of SPPs in complicated environments. Here, we report the utilization of rare-earth-doped nanoparticles to quantitatively upconvert invisible, evanescently confined SPPs into visible photoluminescence emissions for direct far-field visualization of SPPs in a complicated environment. The observed interference fringes between the SPPs and the coherent incident light at the metal surface provide a quantitative measurement of the SPP wavelength and the SPP propagating length and the local dielectric environments. It thus creates a new signaling pathway to sensitively transduce the local dielectric environment change into interference periodicity variation, enabling a new design of directly measurable, spectrometer-free optical rulers for rapid, ultrasensitive label-free detection of various biomolecules, including streptavidin and prostate-specific antigen, down to the femtomolar level.
\end{abstract}

\section{Introduction}

Surface plasmons are collective oscillations of electrons in conductors that confine electromagnetic field at the surface [1-6]. Direct visualization of surface plasmon polaritons (SPPs) and determination of SPP parameters are important and necessary for both fundamental studies and potential applications, including plasmonic waveguides [7, 8], antennas $[9,10]$, sensors [11-13], photovoltaics [14], and metamaterials [15-17]. However, the evanescently confined SPP fields cannot be directly observed with conventional far-field optical methods [3]. Near-field scanning optical microscopy (NSOM) is typically required for monitoring dispersion, propagation, and interference properties of SPPs [18-24]. However, NSOM protocols require flat and dry surfaces and cannot work on plasmonic resonators with biomolecule coverages or liquid environments [3]. The scanning probe method also prevents rapid, high-throughput mapping SPPs over a large area. An alternative approach to map SPPs is to use nanometerscale photoluminescence (PL) probes that can be directly placed in the evanescent tail of the SPP field and excited by SPPs through spectrum coupling, in which the local field intensity is directly transduced into the emission intensity of the PL probes $[3,25]$.

Here, we report the utilization of rare-earth-doped (RE-doped) upconversion nanoparticles (UCNPs) [26, 27] as a unique class of optical probes that can quantitatively "upconvert" SPPs into visible PL emissions, rendering far-field PL images directly mapping the spatial dispersion 
of SPPs with high fidelity for the first time. The SPPs on $\mathrm{Au}$ patterns generated by a 980 or $808 \mathrm{~nm}$ laser can excite the UCNPs located in the evanescent tail of the SPP field. As a result, the spatial distribution of the local SPP field could be effectively converted into PL and mapped by UCNP PL emission intensity. This approach enables a highly convenient yet powerful method for probing the excitation, dispersion, propagation, and interference of SPPs in complicated environments. Notably, the super linear dependence of the upconversion emission intensity $(I)$ on the excitation power $(P)\left(I \propto P^{n}\right.$, where $n$ is the number of photons involved in the energy-transfer-upconversion process for specific emissions) [28, 29] could further enhance the contrast of local field modulation in SPPs. Additionally, other advantages of UCNPs, including NIR excitation, widely tunable emissions, high stability, large anti-Stokes shift, and no photobleaching or blinking effects under high field intensity $[26,27,30]$, make them ideal for the quantitative analysis of SPPs and their dependence on local environments. Significantly, the integration of SPP with UCPL creates a new signaling pathway to sensitively transduce local dielectric environment change into interference periodicity variation, enabling a new design of directly measurable, spectrometer-free optical rulers for ultrasensitive label-free detection of various biomolecules down to the fM level.

\section{Results and Discussion}

2.1. Far-Field Upconversion Visualization of Surface Plasmon Polaritons. UCNPs were prepared through a solution process [28]. Typically, $\beta-\mathrm{NaYF}_{4}: \mathrm{Yb}, \mathrm{Tm}$ and $\beta-\mathrm{NaYF}_{4}: \mathrm{Yb}, \mathrm{Er}$ NPs can give a blue/red and green/red emission, respectively (Fig. S1 in the Electronic Supplementary Material $(\mathrm{ESM})$ ). The particle sizes are about $55 \mathrm{~nm}$ (Figs. S1 and S2 in the ESM) to ensure that they are well within the evanescent tail of the SPPs. Two-dimensional (2D) Au patterns with flat surfaces and specifically designed shapes were lithographically patterned on $\mathrm{SiO}_{2} / \mathrm{Si}$ substrates (Figure 1(a) and Fig. S2 in the ESM). UCNPs were subsequently spin-coated onto the Au patterns to form a randomly dispersed NP layer with a surface coverage of about 30\% (Fig. S2c in the ESM). UCPL was excited by an obliquely shed NIR laser $\left(\lambda_{0}=980 \mathrm{~nm}\right.$, TM mode) and directly imaged by an optical microscope with a CCD camera (Fig. S3 in the ESM).

Systematically dispersed fringes can be observed on all Au patterns with various shapes (Figure 1). All fringes are dispersed periodically along the $x$-direction (horizontal direction), which is also the propagation direction of SPPs. Each stripe mimics the shape of the front edge of the respective Au pattern (Figures 1(c)-1(f)). For Au squares or triangles with a straight edge that is perpendicular to the incident light, the fringes show periodic vertical stripes that are parallel to the front edge (Figures 1(c) and 1(d)). For Au disks or rings, the stripes of the fringes follow exactly the curvature of the front edges (Figures 1(e) and 1(f)). Fringes can be also visualized in different PL color channels by using a mixture of $\beta-\mathrm{NaYF}_{4}: \mathrm{Yb}, \mathrm{Tm}$ and $\beta-\mathrm{NaYF}_{4}: \mathrm{Yb}, \mathrm{Er}$
NPs. The fringes probed by the blue (Figure $1(\mathrm{~g})$ ), green (Figure 1(h)), and red emission (Figure 1(i)) overlap with each other with essentially the same periodicity (Figure $1(j)$ ). In addition, the fringes are strongly dependent on the polarization direction of the incident laser; the strongest fringes can be observed under the TM mode laser excitation, while no interference fringes can be observed when a TE mode laser is used (Fig. S4 in the ESM). Together, these observations indicate that the fringe dispersion is not dependent on the PL emissions, but an intrinsic feature of the incident light and/or SPPs supported on Au patterns.

2.2. Interference Fringes between SPPs and Coherent Incident Light. The above observations represent the first report of far-field optical visualization of such periodic fringes formed by the interference between the SPPs and the coherent incident light, which can only be observed by NSOM [24, 31] or photoemission electron microscopy $[32,33]$ previously. As shown in Figure 2(a), the $d$-spacing of the fringe increases considerably with increasing incident angle. In general, as illustrated in Figure 2(c), when a TM mode laser with the free-space wavelength of $\lambda_{0}$ was obliquely shed onto the $\mathrm{Au}$ patterns with an incident angle of $\theta\left(0<\theta<90^{\circ}\right)$, an SPP wave could be excited at the front edge [32] and propagates along the $x$-direction with the wavelength of $\lambda_{\mathrm{S}}=\lambda_{0} / n_{\mathrm{eff}}$, where $n_{\text {eff }}=\sqrt{\left(\varepsilon_{\mathrm{Au}} \varepsilon_{d}\right) /\left(\varepsilon_{\mathrm{Au}}+\varepsilon_{d}\right)}$ is the effective index and $\varepsilon_{\mathrm{Au}}$ and $\varepsilon_{d}$ are the complex dielectric constant for $\mathrm{Au}$ and dielectrics, respectively $[1,3]$. Meanwhile, the projection of the coherent incident laser at the surface can be considered as a running wave of polarization (RWP) [24] whose amplitude varies sinusoidally along the $x$-direction with the wavelength of $\lambda_{\mathrm{R}}=\lambda_{0} / \sin \theta$. Note that $n_{\text {eff }}>1, \sin \theta<1$, and thus $\lambda_{\mathrm{S}}<\lambda_{0}<\lambda_{\mathrm{R}}$. At the front edge of Au pattern $(x=0)$, both SPP and RWP waves share the same angular frequency and initial phase, which is determined by the incident light. The interference between these two coherent waves with distinct wavelengths will create a localized electric-field oscillation defined by

$$
\begin{aligned}
E_{x}(x, t)= & E_{\mathrm{S} x, 0} e^{-x /(2 L)} \sin \left(k_{\mathrm{S} x} x+\omega_{0} t+\varphi_{0}\right) \\
& +E_{\mathrm{R}} \sin \left(k_{\mathrm{R}} x+\omega_{0} t+\varphi_{0}\right),
\end{aligned}
$$

where $k_{\mathrm{S} x}$ and $k_{\mathrm{R}}$ are the wave vectors for SPPs and RWP, $L$ is the propagation length of SPPs, $E_{\mathrm{S} x, 0}$ is the initial amplitude for SPPs at $x=0, E_{\mathrm{R}}$ is the amplitude for RWP, and $\varphi_{0}$ is the initial phase [21]. The optical nearfield intensity dispersion for the interference fringes along the $x$-direction is

$$
I_{x}=I_{\mathrm{S} x, 0} e^{-x / L}+2 \sqrt{I_{\mathrm{S} x, 0} I_{\mathrm{R}}} e^{-x /(2 L)} \cos \left[\left(k_{\mathrm{S} x}-k_{\mathrm{R}}\right) x\right]+I_{\mathrm{R}},
$$

where $I_{S x, 0}=\left(E_{S x, 0}\right)^{2}$ is the initial intensity for the SPPs at the front edge $(x=0)$, and $I_{\mathrm{R}}=\left(E_{\mathrm{R}}\right)^{2}$ is the intensity of the RWP, which can be considered as a constant since the incident beam is evenly shed on the whole $2 \mathrm{D}$ pattern. Note that the intensity dispersion function is time-independent, allowing the direct observation of steady interference fringes on $\mathrm{Au}$ patterns (Figures 1(b)-1(j)). By 


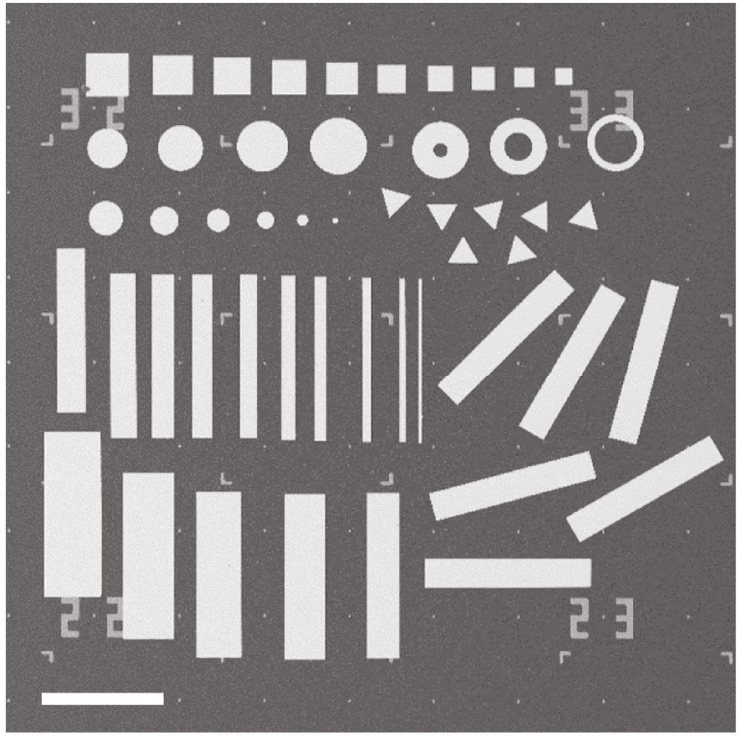

(a)

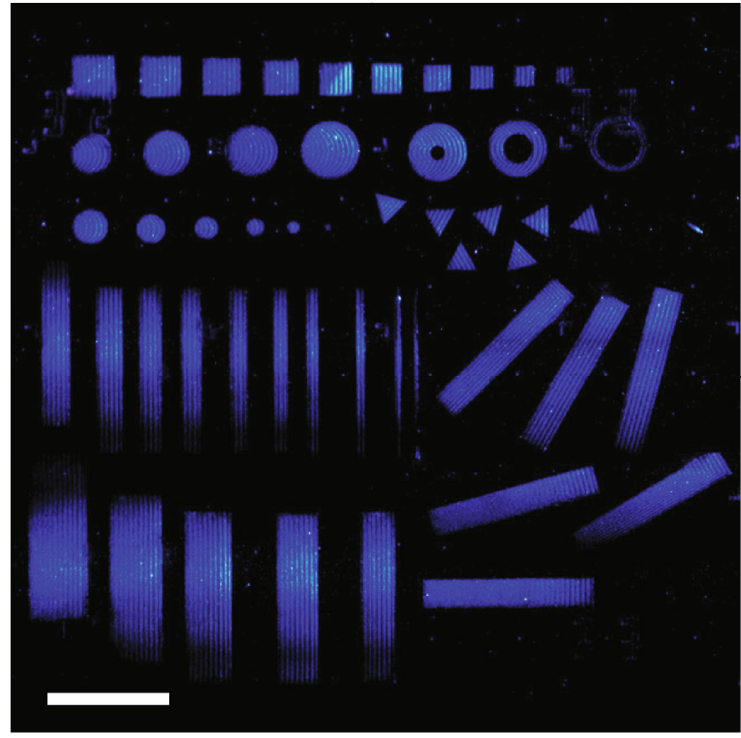

(b)

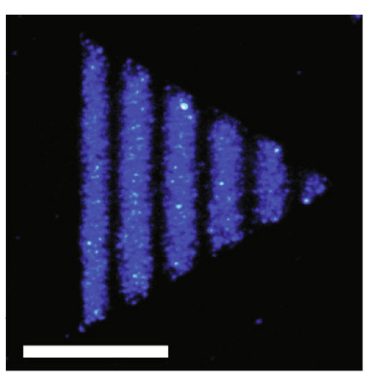

(c)

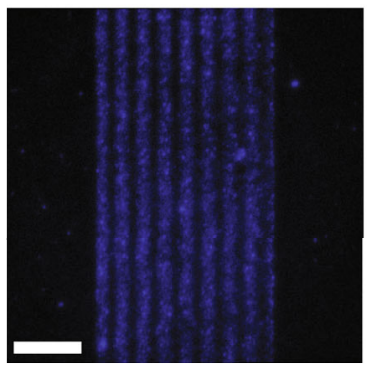

(g)

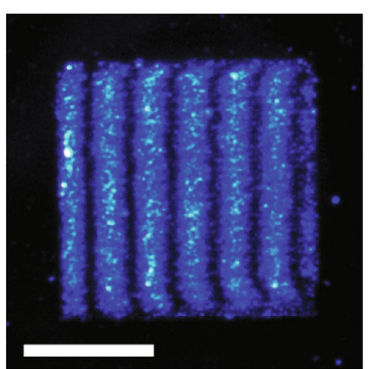

(d)

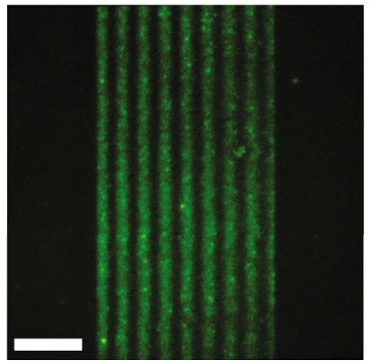

(h)

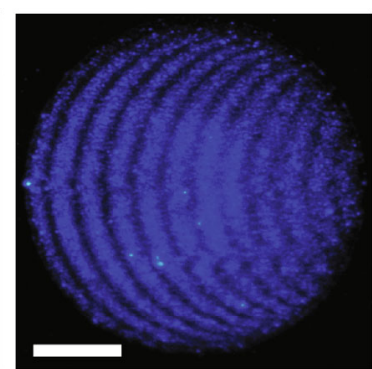

(e)

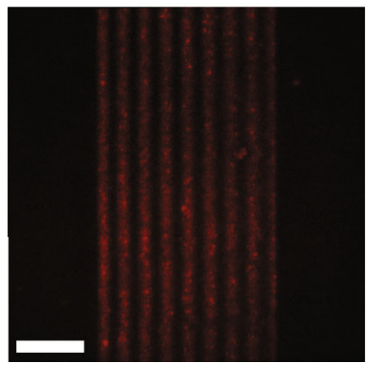

(i)

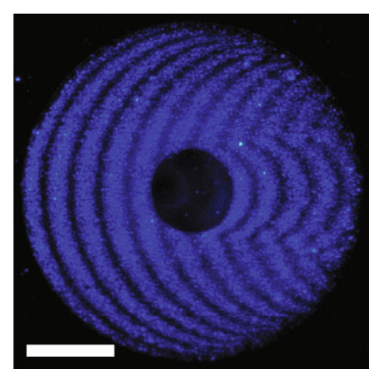

(f)

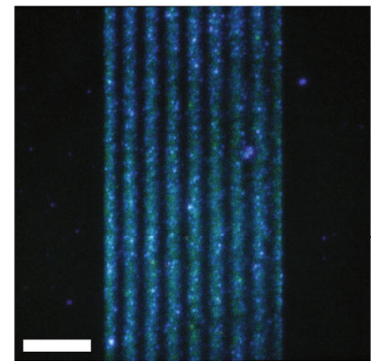

(j)

FIGURE 1: Direct mapping of surface plasmon polaritons (SPPs) by rare-earth-doped (RE-doped) upconversion nanoparticles (UCNPs). (a) SEM image of Au patterns ( $75 \mathrm{~nm}$ in thickness) on $\mathrm{SiO}_{2} / \mathrm{Si}$ substrate $\left(\mathrm{SiO}_{2}\right.$ thickness: $\left.300 \mathrm{~nm}\right)$. (b) Collaged real-color PL image showing interference fringes supported on Au-UCNP patterns with various morphologies (squares, rectangles, triangles, disks, and rings), sizes, and orientations. (c-f) PL images showing interference fringes supported on (c) Au triangle, (d) square, (e) disk, and (f) ring. (g-j) PL images acquired from (g) blue, (h) green, (i) red, and (j) merged channels showing interference fringes probed by UCNPs with different emission bands. The incident light for panel $(\mathrm{b}-\mathrm{j})$ is a TM mode $980 \mathrm{~nm}$ laser with an incident angle of $60^{\circ}$. Scale bar: $(\mathrm{a}, \mathrm{b}) 200 \mu \mathrm{m}$ and (c-j) $25 \mu \mathrm{m}$.

substituting the definition of the wave vectors into equation (2), we can obtain

$I_{x}=I_{\mathrm{S} x, 0} e^{-x / L}+I_{\mathrm{R}}+2 \sqrt{I_{\mathrm{S} x, 0} I_{\mathrm{R}}} e^{-x /(2 L)} \cos \left[\frac{2 \pi}{\lambda_{0}}\left(n_{\mathrm{eff}}-\sin \theta\right) x\right]$.
By assuming $L \rightarrow+\infty$ and $I_{\mathrm{S} x, 0} / I_{\mathrm{R}}=1$ for simplification, the periodic intensity modulation along the $x$-direction can be readily simulated, with a nearly perfect match with the experimental interference fringes observed under different incident angles (Figures 2(a) and $2(\mathrm{~b}))$. 


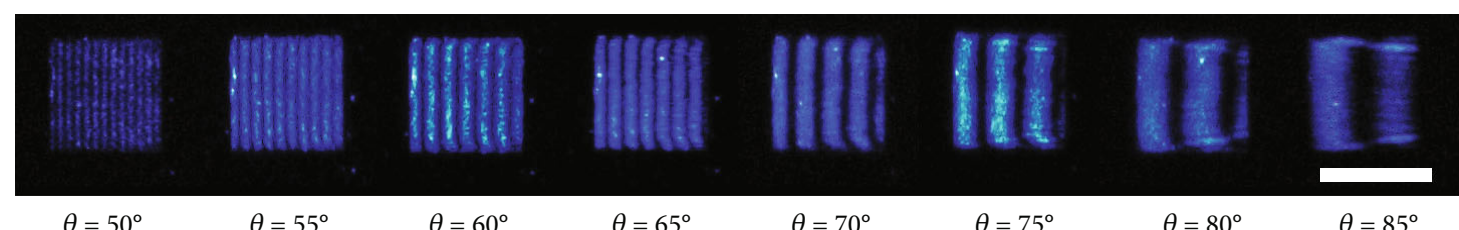

(a)

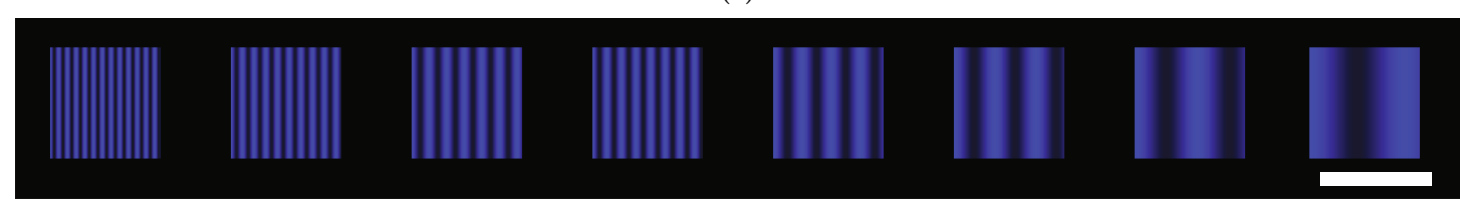

(b)

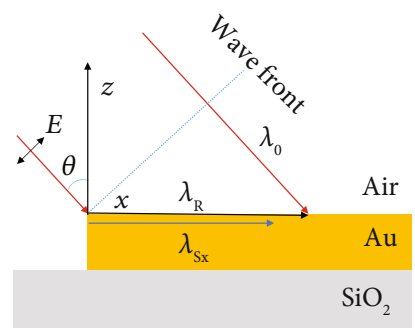

(c)

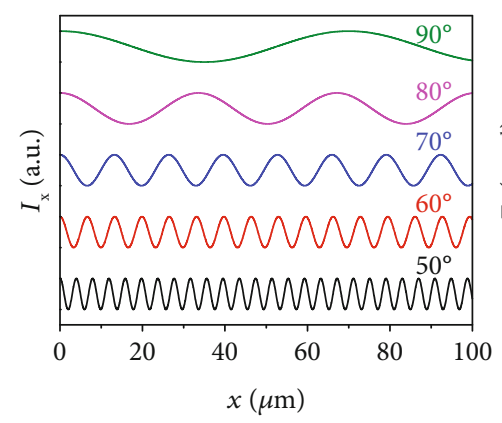

(d)

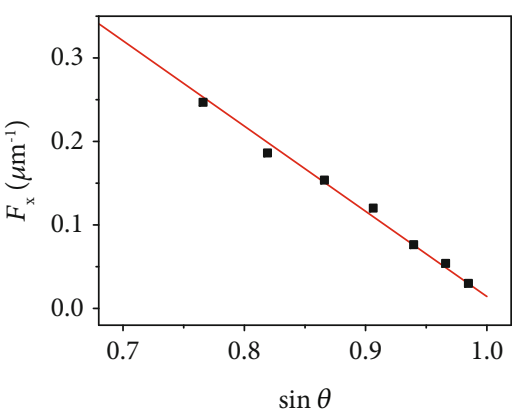

(e)

FIGURE 2: Interference fringes dispersed along the $x$-direction on Au squares with different incident angles. (a, b) Experimentally observed (a) and simulated (b) interference fringes supported by a $(50 \times 50) \mu \mathrm{m}^{2}$ Au square with different incident angles. (c) Schematic illustration for the excitation of SPPs on Au surface by an oblique TM laser. The incident light creates SPPs and a running wave of polarization (RWP). The interference between the SPP and the RWP forms fringes dispersed along the $x$-direction. (d) Simulated intensity dispersion of interference along the $x$-direction for different incident angles. (e) Simulated (red line) and experimentally observed (black square) spatial frequencies $\left(F_{x}\right)$ for various incident angles. The simulated fringes reproduce the experiment results precisely. Scale bar: $(\mathrm{a}, \mathrm{b}) 50 \mu \mathrm{m}$.

According to equation (3), the spatial period ( $d$-spacing) and frequency for the interference fringes along the $x$-direction are defined by

$$
\begin{gathered}
D_{x}=\frac{\lambda_{0}}{n_{\mathrm{eff}}-\sin \theta}, \\
F_{x}=\frac{n_{\mathrm{eff}}-\sin \theta}{\lambda_{0}} .
\end{gathered}
$$

The $d$-spacing and frequency of the interference fringes are functions of the free-space wavelength, incident angle, and effective index, suggesting that the period of the interference fringes increases with the increasing incident angle (Figure 2(d)). The modulation frequency $F_{x}$ and $\sin \theta$ exhibit a linear relationship (Figure 2(e)), which is further confirmed by experimental observations, demonstrating that the proposed model can provide a sound foundation for further understanding and modulation of the SPP dispersion, propagation, and interference.
2.3. Attenuation of SPPs along the Propagation Direction. In addition to periodic modulation, it is also apparent that the overall UCPL intensity attenuates along the propagating direction on a long Au stripe (Figures 3(a) and 3(b)). Plots of the intensity dispersion along the $x$-direction under the incident angles of 70 and $60^{\circ}$ show clearly periodic modulation and gradual attenuation of the modulation amplitude (black lines in Figures 3(e) and 3(f)). To quantitatively analyze the intensity dispersions, we need to consider the attenuation effects of SPPs and the initial intensity ratio between SPPs and RWP $\left(I_{\mathrm{S} x, 0} / I_{\mathrm{R}}\right)$, which is determined by the excitation efficiency for SPPs. Our model provides an accurate pathway to derive the propagation length $(L)$ and intensity ratio $\left(I_{\mathrm{S} x, 0} / I_{\mathrm{R}}\right)$ of SPPs by fitting the interference intensity dispersion with equation (3). Through numerical simulations of the observed interference patterns, we can obtain the two important SPP parameters of $L=(50.3 \pm 2.6) \mu \mathrm{m}$ and $I_{\mathrm{S} x, 0} /$ $I_{\mathrm{R}}=0.04$ when $\theta=70^{\circ}$ (Figure $3(\mathrm{e})$ ) and $L=(47.3 \pm 2.5)$ $\mu \mathrm{m}$ and $I_{\mathrm{S} x, 0} / I_{\mathrm{R}}=0.015$ when $\theta=60^{\circ}$ (Figure 3(f)). Such results reveal that the propagation length values on the $\mathrm{Au}$ stripes are independent on the incident angle of the 


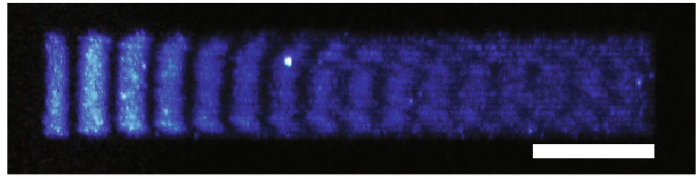

(a)

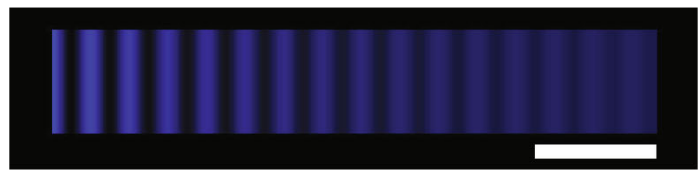

(c)

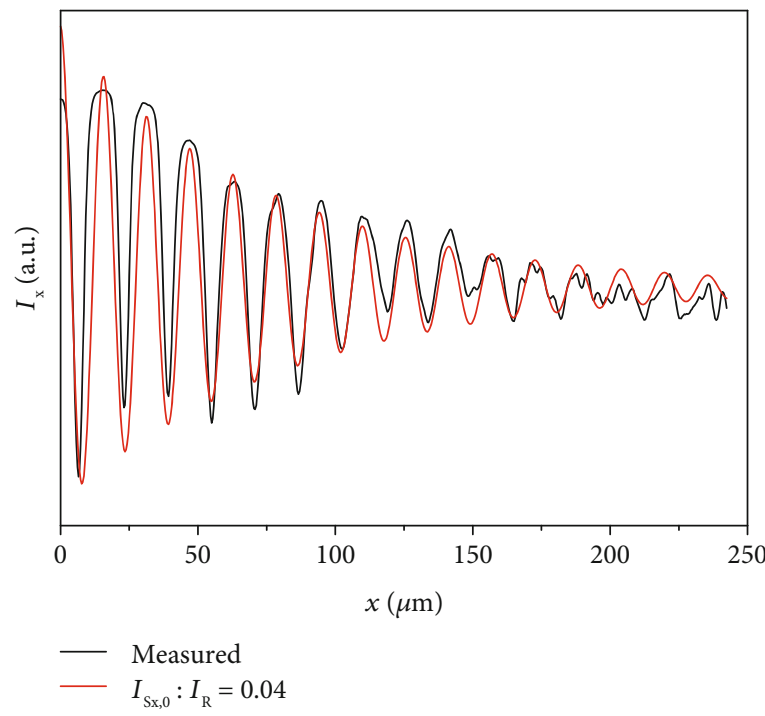

(e)

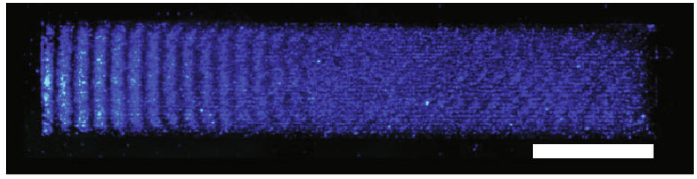

(b)

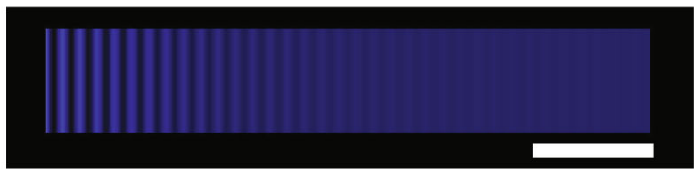

(d)

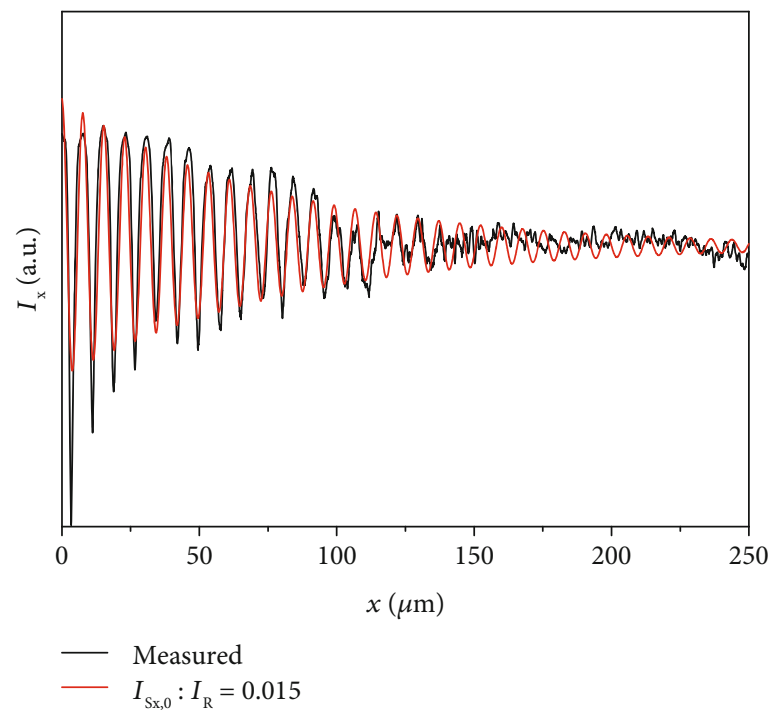

(f)

Figure 3: Attenuation of SPPs along the propagation direction. (a, b) PL images for the interference fringes along the $x$-direction on a Au stripe with the incident angle of (a) $70^{\circ}$ and (b) $60^{\circ}$, respectively. (c, d) Simulated patterns corresponding to the interference fringes shown in (a) and (b), respectively. (e, f) Experimentally measured (black) and simulated (red) intensity dispersion for the interference fringes show in (a, c) and (b, d), respectively. $\lambda_{0}=980 \mathrm{~nm}$. Scale bar: (a-d) $50 \mu \mathrm{m}$.

laser and are comparable to the theoretical and experimental results measured through NSOM previously $[1,3$, $7,20,24]$. With these fitting parameters, the $2 \mathrm{D}$ intensity distribution image can be regenerated (Figures 3(c) and $3(\mathrm{~d})$ ), which match well with the experimental UCPL images (Figures 3(a) and 3(b)). In accordance with previous experimental and theoretical results [31, 34], our observations prove that the propagation length shows no statistically significant differences at various incident angles (Fig. S5b in the ESM) [3]. However, the SPP excitation efficiency shows a significant polarization sensitivity [35]. At higher incident angle, the component of the incident light polarized perpendicularly to the Au surface becomes larger so that higher SPP excitation efficiency can be achieved (Fig. S5c in the ESM). Compared to previous methods heavily relying on the invasive and complicated NSOM protocols, our upconversion strategy proves to be a simple yet accurate far-field approach to obtain the key parameters, the attenuation length, and the excitation efficiency of SPPs.
2.4. Interference Fringes on $\mathrm{Au}$ Patterns with Various $2 D$ Morphologies. Another important parameter for the excitation and propagation of SPPs is the azimuthal angle of the incident light. As indicated by Figure 4(a), SPPs can be excited at each point $\left(x_{i}, y_{i}\right)$ on the two edges of a rotated Au square and propagate along the $x$-direction. For each horizontal line $\left(y=y_{i}\right)$, the interference between SPPs and RWP still follows equations (1) and (2). Therefore, the overall interference fringes on a rotated pattern can be obtained by integrating the intensity dispersion along each horizontal line, that is,

$$
\begin{aligned}
I_{x}\left(y=y_{i}\right)= & I_{\mathrm{S} x, 0} e^{-x / L}+2 \sqrt{I_{\mathrm{S} x, 0} I_{\mathrm{R}}} e^{-x /(2 L)} \\
& \cdot \cos \left[\left(k_{\mathrm{S} x}-k_{\mathrm{R}}\right)\left(x-x_{i}\right)\right]+I_{\mathrm{R}},
\end{aligned}
$$

where $x_{i}$ is the $x$-coordinate of the starting point at the front edge for each $y=y_{i}$ line. According to equation (6), we can predict that for Au patterns with any 2D morphologies, the $x$-direction interference fringes will exactly inherit the shapes 


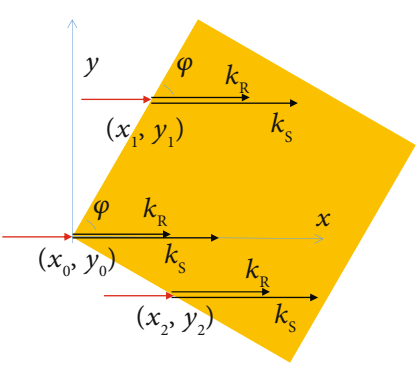

(a)
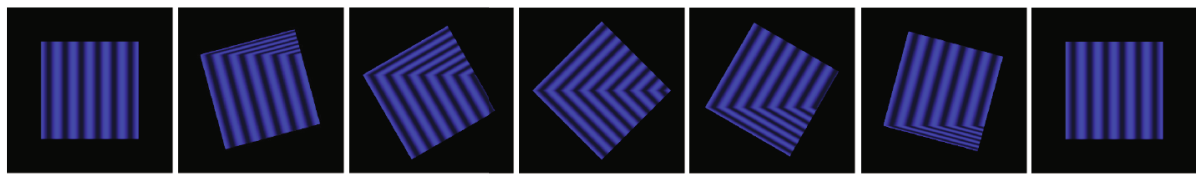

(c)
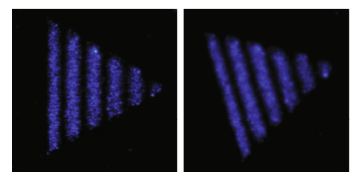

$\varphi=15^{\circ}$

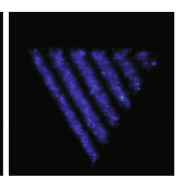

$\varphi=30^{\circ}$

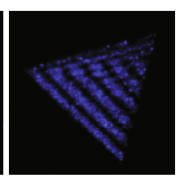

$\varphi=45^{\circ}$

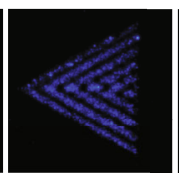

$\varphi=60^{\circ}$

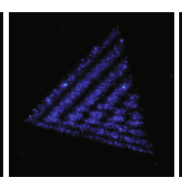

$\varphi=75^{\circ}$

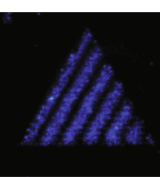

$\varphi=90^{\circ}$

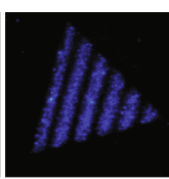

$\varphi=105^{\circ}$

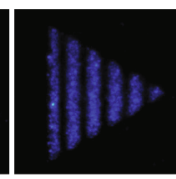

(d)
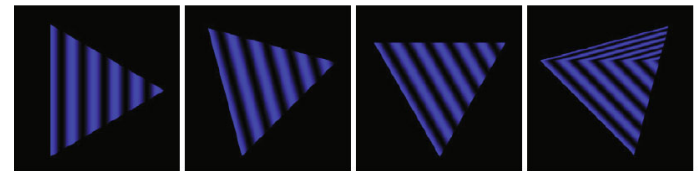
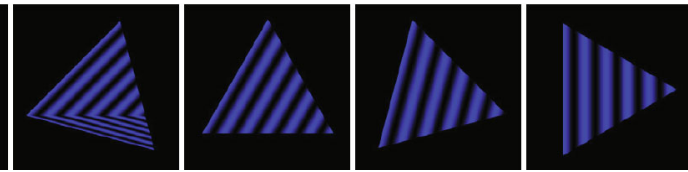

(e)

FIGURE 4: Interference fringes supported on Au patterns with various orientations. (a) Schematic illustration for the interference between SPPs and RWP on a rotated Au square with an azimuthal angle of $\varphi$. (b, c) Experimentally observed (b) and theoretically simulated (c) interference fringes supported on a $(50 \times 50) \mu \mathrm{m}^{2}$ Au square with different rotation angles. (d, e) Experimentally observed $(\mathrm{d})$ and simulated (e) interference fringes on a rotated Au triangle (edge length: $50 \mu \mathrm{m}$ ). $\theta=60^{\circ}$.

of the front edges where SPPs are stimulated. As shown in Figures $4(\mathrm{~b})$ and $4(\mathrm{c})$, the observed and simulated fringes on a rotated Au square fit each other well for all azimuthal angles. This principle stands for Au patterns with all other morphologies, including triangles (Figures $4(\mathrm{~d})$ and $4(\mathrm{e})$ ), disks (Figure 1(e)), rings (Figure 1(f)), and even irregular patterns (Fig. S6 in the ESM).

\subsection{Superposition of Vertical and Lateral Interference Fringes.} The Au patterns can also support a series of lateral fringes with much smaller spatial periods (Figs. S7-9 in the ESM). Previous theoretical simulations suggest that narrow metal stripes can support several modes of lateral SPPs [36]. Our studies show that clear lateral fringes can be observed when two parallel lateral edges of Au patterns are simultaneously illuminated by the incident beam (Fig. S7 in the ESM), providing a direct evidence for the strong lateral confinement effect on SPPs supported by metal stripes with finite width (Fig. S8 in the ESM) [36]. The total intensity dispersion for the interference fringes is the superposition of the $x$-direction interference between SPPs and RWP and the $y$-direction interferences between two SPP waves travelling in opposite directions (Fig. S9 in the ESM). The fitted interference fringes reproduce the observed results with high fidelity, demonstrating that UCNPs can function as effective optical probes for directly visualizing the excitation, dispersion, propagation, and interference properties of SPPs.

Together, UCNPs can upconvert the invisible, evanescently confined SPP fields into visible PL images, allowing far-field visualizing the interference fringes formed by SPPs and the coherent incident light. UCNPs represent a novel kind of PL probes to reveal the intrinsic dependence of SPP excitation and propagation on several basic factors $\left(\lambda_{0}, \theta, \varphi\right.$, $\varepsilon_{m}$, and $\left.\varepsilon_{d}\right)$ in complicated environments. Our method provides a facile far-field approach for determining the key SPP parameters (excitation efficiency, propagation length), which can play a critical and indispensable role in SPPrelated research and practices.

\subsection{Interference Fringes as Ultrasensitive Spectrometer-Free} Optical Ruler. Besides the significance in fundamental SPP research, the interference fringes can be also explored as an optical sensor to monitor the variations in local dielectric environment, according to equation (4). The interference fringes show a rapid and reversible response to local dielectric change that can be monitored in real-time (movie S1 in the ESM). The linear relationship between the frequency of the fringes and the effective index (equation (5)) is confirmed by experiment results over large effective index variations (Fig. S10 and Table S1 in the ESM). In principle, the lowest 


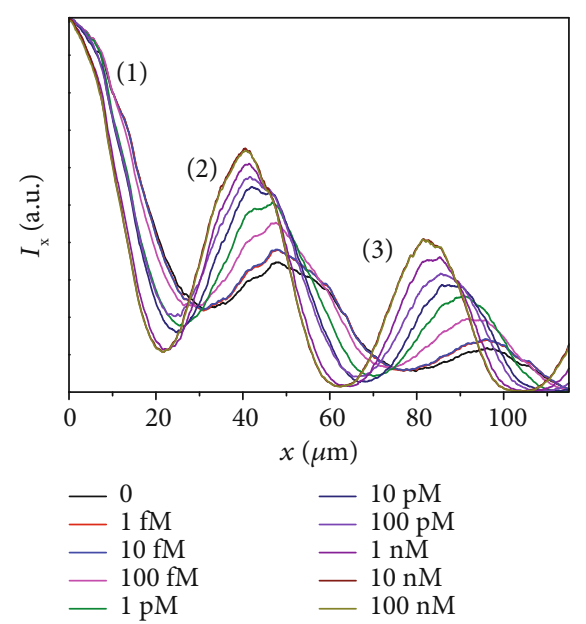

(a)
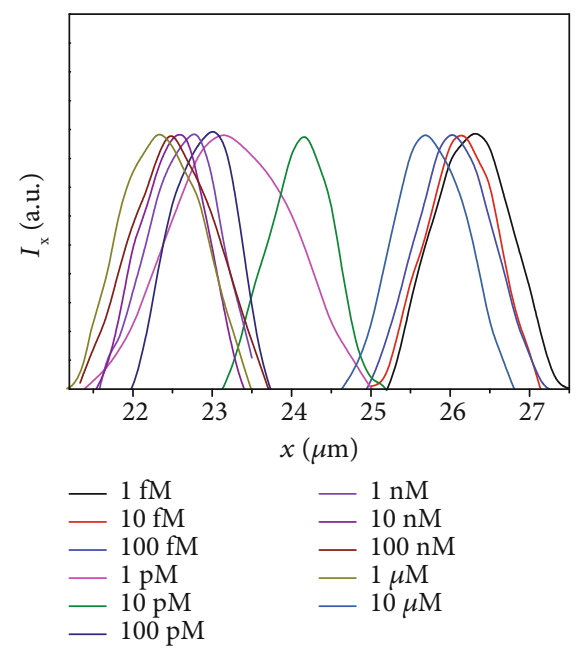

(c)

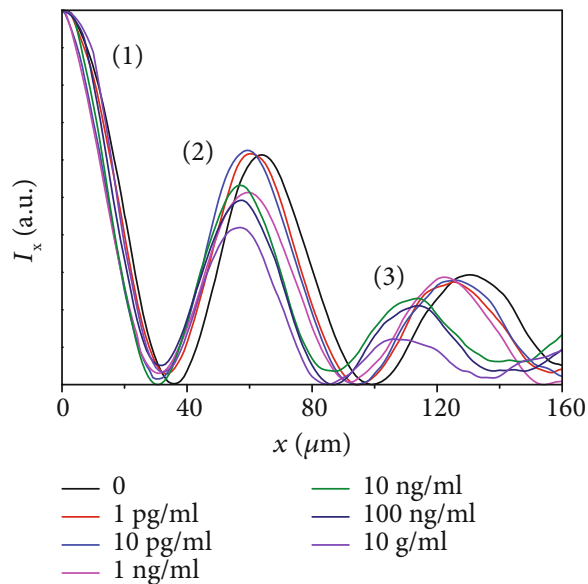

(e)

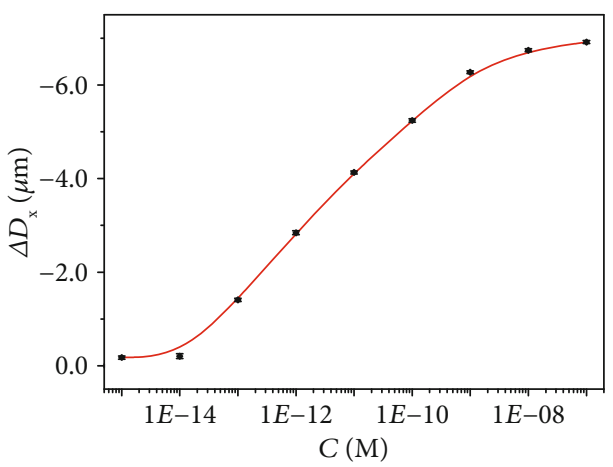

(b)

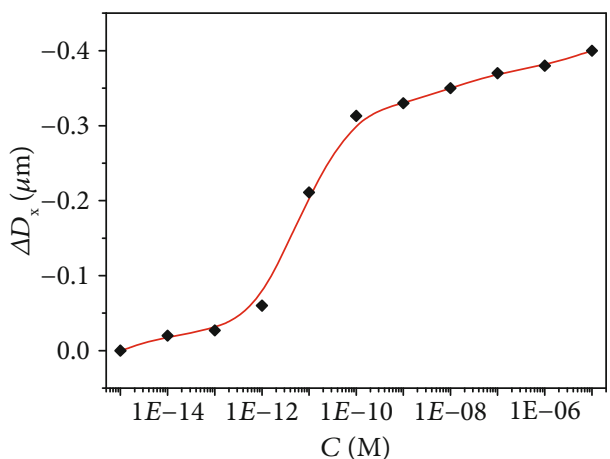

(d)

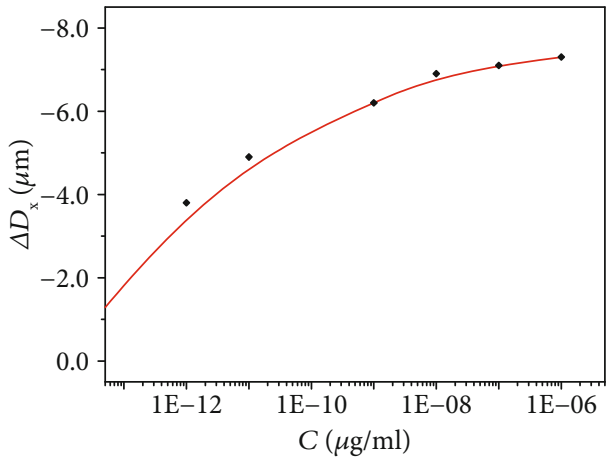

(f)

FIGURE 5: Response to streptavidin and prostate-specific antigen (PSA) of different concentrations for specifically modified Au-UCNP sensors. (a) Normalized intensity distribution of the interference fringes acquired in air after immersing the biotinylated Au-UCNP pattern in streptavidin solution of different concentrations. (b) Variation of $\Delta D_{x}$ (in air) versus streptavidin concentrations for the biotinylated Au-UCNP pattern in panel (a). (c) Normalized intensity distribution of the $10^{\text {th }}$ interference fringe acquired in situ when the biotinylated Au-UCNP pattern is immersed in streptavidin solution of different concentrations. (d) Variation of $\Delta D_{x}$ (in solution) versus streptavidin concentrations for the biotinylated Au-UCNP pattern in panel (c). (e) Normalized intensity distribution of the interference fringes dispersed along the $x$-direction acquired in $\mathrm{N}_{2}$ after the antibody-modified Au-UCNP sensor is exposed to PSA solution of different concentrations. (f) Variation of $\Delta D_{x}$ versus PSA concentrations for the antibody-modified Au pattern. 
detectable refractive index variation can be defined by dividing the resolution of the far-field microscope by the refractive index sensitivity of the interference fringes. On the one hand, the $d$-spacing and the refractive index sensitivity for the interference fringes can be increased dramatically by increasing the incident angle (Fig. S11 in the ESM) and incident wavelength (Fig. S12 in the ESM). In particular, equation (4) reveals that the refractive index sensitivity can be enhanced significantly when $\left(n_{\text {eff }}-\sin \theta\right) \rightarrow 0$. The refractive index sensitivity can be over $3000 \mu \mathrm{m}$ per refractive index unit (RIU) in air with the incident angle of $85^{\circ}$ (Fig. S11 in the ESM). On the other hand, the resolution of the far-field optical microscope can also be enhanced by using short wavelength emissions as optical signal enabled by the UCPL (e.g., $<500 \mathrm{~nm}$ ). According to the Abbe diffraction limit [37], a 100x objective with the numerical aperture of 0.80 has the spatial resolution of about $300 \mathrm{~nm}$ for the working wavelength of $476 \mathrm{~nm}$ (blue emission of $\beta$ $\mathrm{NaYF}_{4}: \mathrm{Yb}, \mathrm{Tm}$ NPs, Fig. $\mathrm{S} 1$ in the ESM). Therefore, a variation of $10^{-4}$ RIU can be detected when a single period of the interference fringes is measured. And a variation of $10^{-5}$ RIU (or even lower) can be monitored when ten or more periodically dispersed fringes were measured to obtain the average spatial period value. That is, the refractive index sensitivity of our SPP-UCPL sensors is comparable to that of complex plasmonic metamaterial sensors working in the NIR regime [11, 38]. Moreover, as compared to previous plasmonic sensors that measure the wavelength shift in response to refractive index variation in the NIR regime $[11,12,38,39]$, our approach can directly transduce the local dielectric environment changes into the variations in interference fringe periodicity, thus enabling an entirely new design of ultrasensitive spectrometer-free optical ruler for direct detection of various molecular binding events in real time.

A typical biotin-streptavidin affinity model $[11,38,39]$ was first employed to evaluate the sensitivity and limit of detection (LOD) of this unique spectrometer-free optical ruler. In principle, the specific binding of streptavidin to surface biotin groups can induce an increase in a local effective index, which can, in turn, lead to a decrease in the interference fringe $d$-spacing. Figures $5(\mathrm{a})$ and $5(\mathrm{~b})$ and Fig. S13 in the ESM show the variation of the intensity dispersion for the interference fringes acquired in air after the biotinylated sensor was exposed to streptavidin solutions of different concentrations through a microfluidic configuration (Fig. S14 in the ESM). The peaks for intensity dispersion curves show a significant left-shift with increasing streptavidin concentration (Figure 5(a)). The variation of $d$-spacing versus the streptavidin concentration (Figure 5(b)) indicates that the LOD for streptavidin can be as low as $10 \mathrm{fM}$ for the single-pattern SPPUCPL sensor. Additionally, our direct measurement in solution reveal that streptavidin of the concentration at the pM level can be detected by the SPP-UCPL optical ruler in real-time (Figures 5(c) and 5(d) and Fig. S15 in the ESM). To our knowledge, previously reported LOD values to streptavidin in a wavelength-shift type of plas- monic sensors typically range from $\mathrm{pM}$ to $\mu \mathrm{M}$ concentrations [40], requiring the help of spectrometers or other scanning components [41]. Therefore, the SPP interference fringes represent a new design of ultrasensitive, directly measurable optical rulers for biomolecule detection.

To demonstrate its broad applicability and capability, we have further employed the SPP-UCPL optical ruler for highly sensitive detection of another important biomarker, prostate-specific antigen (PSA) [42]. Most of the current PSA biosensors are derived from variations of enzyme-linked immunosorbent assays (ELISA) [43-45], requiring various exogenous label molecules for the detection, which inevitably impedes their applications in both fundamental research and healthcare diagnostics. Other studies usually require high-cost instrument [46, 47], complicated processing protocols such as DNA scanometric detection [48], and/or complex structures of sandwich immunoassay $[49,50]$ to reach a $\mathrm{fM}$ level PSA sensitivity. In contrast, with our most simplified SPP-UCPL optical ruler, a LOD of less than $1 \mathrm{pg} / \mathrm{ml}$ (30 fM) for PSA can be achieved with a simple optical observation and space measurement (Figures 5(e) and 5(f) and Fig. S16 in the ESM), which is comparable to previous reports that require more complicated instrumentation (Table S3 in the ESM). Together, our method based upon SPP-RWP interference fringes represents a cost-effective, directly measurable optical ruler for ultrasensitive, spectrometer-free detection and screening of specific chemical or biochemical species.

\section{Conclusion}

In summary, we have demonstrated the first utilization of RE-doped UCNPs as nanoscale optical probes that can quantitatively upconvert evanescently confined SPPs into visible emissions for real-time mapping SPPs in complicated environments with high throughput and high fidelity. The interference fringes can serve as an ultrasensitive, directly measurable optical ruler for rapid detection of local dielectric variations. A variation of the refractive index $<10^{-5}$ RIU can be detected directly without any bulk spectrometers or scanning components. In practice, the SPP-UCPL sensor shows a LOD about $10 \mathrm{fM}$ for streptavidin and $<30 \mathrm{fM}$ for PSA, respectively. The integration of SPP and UCPL materials opens a new possibility to quantitatively map the SPP dispersion in complicated environments and to design a new generation of ultrasensitive, spectrometer-free sensors. We believe that our findings shall shed light on fundamental studies and applications of SPP and UCPL materials.

\section{Conflicts of Interest}

The authors declare no competing financial interests.

\section{Authors' Contributions}

Anxiang Yin and Hao Jing contributed equally to this work. 


\section{Acknowledgments}

X.D. acknowledge the financial support from the National Science Foundation through grant No. 1610361.

\section{Supplementary Materials}

Supplementary 1. Fig. S1: TEM images and upconversion emission spectra for $\beta-\mathrm{NaYF} 4: \mathrm{Yb}, \mathrm{Tm}$ and $\beta-\mathrm{NaYF} 4: \mathrm{Yb}, \mathrm{Er}$ NPs. (a) TEM image and (b) upconversion emission spectra for $\beta$-NaYF4:Yb,Tm NPs. (c) TEM image and (d) upconversion emission spectra for $\beta-\mathrm{NaYF} 4: \mathrm{Yb}, \mathrm{Er}$ NPs. The average particle size is about $55 \mathrm{~nm}$ for the $\beta-\mathrm{NaYF} 4: \mathrm{Yb}, \mathrm{Tm}$ NPs and $60 \mathrm{~nm}$ for the $\beta-\mathrm{NaYF} 4: \mathrm{Yb}, \mathrm{Er}$ NPs, respectively. When excited by a $980 \mathrm{~nm}$ continues wave diode laser, the $\beta-\mathrm{NaY}$ F4:Yb,Tm NPs have a blue/red emission, while the $\beta-\mathrm{NaY}$ $\mathrm{F} 4: \mathrm{Yb}, \mathrm{Er}$ NPs give a green/red emission. The corresponding $\mathrm{f}-\mathrm{f}$ transitions for each emission are identified in panels (b) and (d). Scale bar: (a) $200 \mathrm{~nm}$, (c) $100 \mathrm{~nm}$. Fig. S2: AFM profiles for the Au patterns and Au-UCNP composites. (a) AFM profile of bare Au surface, showing a surface roughness of $0.8 \mathrm{~nm}$. (b) Large area AFM profile showing the edge of the Au-UCNP pattern. Note that there is a single layer of UCNPs close to the edge of the Au pattern. The size of the UCNP is about $55 \mathrm{~nm}$, which is consistent with the TEM observations, and the height of the Au pattern is about $75 \mathrm{~nm}$. (c) AFM image of UCNPs supported on the Au pattern, showing a randomly dispersed NP single-layer with a surface coverage of about 30\%. (d) Enlarged AFM image of the UCNPs on Au surface, showing the size and shape uniformity for the UCNPs. Scale bar: (a, c) $1 \mu \mathrm{m}$, (b) $2 \mu \mathrm{m}$, and (d) $100 \mathrm{~nm}$. Fig. S3: schematic illustration of the set-up for the observation of fringes formed by the interference between the incident light and the SPPs. The Au-UCNP pattern supported on a $\mathrm{SiO}_{2} / \mathrm{Si}$ substrate is fixed on the specimen holder of the optical microscope. A $980 \mathrm{~nm}$ continuous wave diode laser is obliquely shed onto the sample, and the upconversion emission is collected by the objective $(10 \mathrm{x}, \mathrm{NA}=0.25 ; 50 \mathrm{x}$, $\mathrm{NA}=0.50$; and $100 \mathrm{x}, \mathrm{NA}=0.8$ LMPLFLN-BD objectives). The polarization direction of the incident beam is controlled by a polarizer and a half-wave plate. Fig. S4: interference fringes observed with different polarization directions of the incident laser. No interference patterns can be observed when the laser is in the TE mode, while the strongest interference fringes are generated by the TM mode incident light. The intensity of the incident light and exposure parameters were fixed during the whole test. Note that the intensity of the blue spot at the left-up corner in each panel, which is located on the $\mathrm{SiO}_{2} / \mathrm{Si}$ substrate far away from the Au pattern, remains constant and can be used as a reference for the intensity change of the interference fringes. Scale bar: $25 \mu \mathrm{m}$. Fig. S5: interference intensity dispersion observed at different incident angles. (a) Experimentally measured intensity dispersion of interference fringes along the $x$-direction on a $\mathrm{Au}$ stripe at different incident angles. (b) The fitted SPP propagation length versus the incident angle curve, showing no statistically significant variation among the propagation length values at different incident angles. (c) Relative intensity ratios (fitted) between the initial SPP and the RWP
$\left(I_{\mathrm{S} x, 0}: I_{\mathrm{R}}\right)$ or the incident light $\left(I_{\mathrm{S} x, 0}: I_{0}\right)$, showing an increase of SPP excitation efficiency with the increase of the incident angle. The intensity of the incident light is calculated by $I_{\mathrm{R}}=I_{0} \cos \theta$. Error bars represent the standard deviations from three independent experiments. Fig. S6: interference fringes supported by an irregular Au pattern. (a) Bright-field image of the irregular Au pattern. (b) PL image for the interference fringes supported on the Au pattern. The shape of the fringes follows the front (right) edges of the Au pattern. Scale bar: $50 \mu \mathrm{m}$. Fig. S7: interference fringes supported on different $\mathrm{Au}$ patterns showing the edge effects on the fringes. (a) Au patterns with several bevel edges, $(b, c)$ L-shape Au pattern, and (d) rectangle $\mathrm{Au}$ pattern. The red dashed line highlights the boundary between different fringes. In panels (a)-(c), no lateral fringes can be observed since there are no, or only one, lateral edge that is excited by the incident light. However, in panel (d), clear lateral fringes are observed because both lateral edges are excited by the incident light to create two SPPs travelling along the opposite direction to form the interference fringes. Scale bar: (a, b) $50 \mu \mathrm{m}$, (c, d) $20 \mu \mathrm{m}$. Fig. S8: interference fringes supported by Au stripes with different widths. (a, b) PL images showing the lateral interference fringes on $\mathrm{Au}$ stripes with the width of $5 \mu \mathrm{m}$ (a) and $50 \mu \mathrm{m}$ (b), respectively. (c) PL image showing both the vertical and lateral interference fringes on $\mathrm{Au}$ stripes with different widths. (d) The $d$ -spacing for the lateral fringes versus the width curve for lateral fringes supported on $\mathrm{Au}$ stripes with different widths, showing the lateral confinement effects when the Au stripe width is smaller than $20 \mu \mathrm{m}$. Scale bar: (a, b) $10 \mu \mathrm{m}$, (c) $50 \mu \mathrm{m}$. Fig. S9: vertical and lateral interference fringes supported on Au stripes. (a, b) Observed (a) and simulated fringes (b) supported on a $50 \mu \mathrm{m}$ wide $\mathrm{Au}$ stripe. (c, d) Corresponding intensity dispersion along the $x$ - and $y$-directions for observed and simulated fringes shown in (a,b). The measured (black) and simulated (red) curves fit each other precisely. $\theta=60^{\circ}$. Scale bar: (a, b) $20 \mu \mathrm{m}$. Fig. S10: response to different solvents. (a) Interference fringe ( $x$-direction) frequency versus effective index (neff) curve for different surrounding media (air, methanol, water, ethanol, isopropanol, 1-butanol, and DMF). (b) PL images for the interference fringes supported by a $50 \mu \mathrm{m}$ wide $\mathrm{Au}$ stripe immersed in different dielectrics. The period for the fringes decreases with increasing the effective index by changing to solvents with higher dielectric constant and recovers after the solvent is evaporated by $\mathrm{N} 2$ flow. The free-space wavelength is $980 \mathrm{~nm}$, and the incident angle is $60^{\circ}$. Fig. S11: calculated $D_{x}-n_{\mathrm{d}}$ relationship at different incident angles. $(\mathrm{a}-\mathrm{c}) D_{x}-n_{\mathrm{d}}$ curves at different incident angles. (d-f) The absolute value of the slope for the $D_{x}-n_{\mathrm{d}}$ curve (i.e., refractive index sensitivity) is plotted for different incident angles, showing that the refractive index sensitivity increases dramatically with the increase of the incident angle. Fig. S12: influence of the incident light wavelength on the periods of the interference fringes. (a) Calculated $d$-spacing (period) values of the $x$-direction interference fringes (if present) versus the free-space wavelength 
curves for different incident angles, showing that the $d$ -spacing will increase at all incident angles when the incident free-space wavelength is increased. $(b, c)$ Interference fringes supported by a $50 \mu \mathrm{m}$ wide Au stripe excited by a (b) $808 \mathrm{~nm}$ or (c) $980 \mathrm{~nm}$ laser with the same incident angle $\left(\sim 75^{\circ}\right)$, confirming the calculation results shown in panel (a). Another type of UCNPs, $\beta$-NaGdF4:Yb,Er@NaGdF4:Nd NPs, which can be excited by either 808 or $980 \mathrm{~nm}$ laser1, were used to probe the intensity dispersion of the interference fringes under incident light with the free-space wavelength of 808 or $980 \mathrm{~nm}$, respectively. Scale bar: $25 \mu \mathrm{m}$. Fig. S13: real-color image of the interference fringes showing the response to streptavidin for biotinylated Au-UCNP sensors. The fringes supported on the biotinylated Au-UCNP pattern show a significant systematic shift (decrease of $d$-spacing) after the binding of streptavidin by surface biotin groups. For the sensing of streptavidin at each bulk concentration, after the binding of surface biotin and streptavidin in the microfluidic channel (illustrated in Fig. S14), the Au-UCNP pattern and the channel are rinsed with DI water for three times to remove excessive unspecifically bound streptavidin molecules and dried under N2 flow. PL images were collected to verify that stable fringes were obtained for the calculation of spatial periods. The three bright stripes highlighted correspond to the three intensity peaks shown in Figure 5(a) in the main text. Scale bar: $50 \mu \mathrm{m}$. Fig. S14: schematic illustration for the microfluidic channels used in the solvent and streptavidin sensing experiments. The biotinylated Au-UCNP pattern supported on a $\mathrm{SiO}_{2} / \mathrm{Si}$ substrate is placed in a PDMS channel with a volume of about 10 $\mu \mathrm{l}$, which is sealed with a cover slide $(0.15 \mathrm{~mm}$ in thickness) and tightened with clamps. The sample is fixed on the specimen holder of the optical microscope. A $980 \mathrm{~nm}$ laser is obliquely shed onto the sample and the upconversion emission is collected by the objective. Different solvents or solutions with different streptavidin concentrations were injected by syringe pumps. N2 flow was used to blow dry the samples before changing different solvents. Fig. S15: response to streptavidin solution of different concentrations for biotinylated Au-UCNP sensors. Directly observed interference fringes supported on the Au-UCNP square immersed in air or aqueous solution of streptavidin with different concentrations. Scale bar: $20 \mu \mathrm{m}$. Fig. S16: real-color image of the interference fringes showing the response to PSA for biotinylated Au-UCNP sensors. The fringes supported on the antibodymodified Au-UCNP pattern show a significant systematic shift (decrease of $d$-spacing) after the binding of PSA by surface antibody groups. For the sensing of PSA at each bulk concentration, after the binding of surface antibody and PSA in the microfluidic channel (illustrated in Fig. S13), the AuUCNP pattern and the channel are rinsed with DI water for three times to remove excessive nonspecifically bound PSA molecules and dried under N2 flow. PL images were collected to verify that stable fringes were obtained for the calculation of spatial periods. The three bright stripes highlighted correspond to the three intensity peaks shown in Figure 5(e) in the main text. Scale bar: $100 \mu \mathrm{m}$. Fig. S17: time-dependent intensity dispersion for the interference between the RWP and the SPPs in one period, showing the envelope curve (low frequency, cyan) and the time-dependent intensity curves (high frequency) for $2 \omega 0 t=0$ (black), $\pi / 2$ (red), $\pi$ (green), and $3 \pi / 2$ (blue), respectively. The incident light beam $(\lambda 0=980 \mathrm{~nm})$ is obliquely shed onto the surface at $\theta=60^{\circ}$. Fig. S18: conversion from UCPL intensity to local field intensity. (a) Emission intensity versus excitation power density curves for the $476 \mathrm{~nm}$ emission of $\beta$-NaYF4:Yb,Tm NPs when excited by a $980 \mathrm{~nm}$ CW laser. The slope for the $\operatorname{Ln}\left(I_{476}\right)-$ $\operatorname{Ln}(P)$ curve is 3.03. (b) Measured UCPL intensity $(476 \mathrm{~nm})$ dispersion, local field intensity converted based on the $I_{\mathrm{LF}} \propto$ $\sqrt[3]{I_{476}}$ relationship, and simulated intensity dispersion curves. Table S1: calculated period of the interference fringe patterns in different solvents. Table S2: calculated period of the interference fringe patterns on air/Au surface with different incident wavelengths and angles. Table S3: comparison of different PSA detection methods.

Supplementary 2. Real-time observation of the interference fringes in response to the local dielectric change. The surrounding environment for the Au-UCNP stripe was changed from air to butanol and then to air again during the observation.

\section{References}

[1] W. L. Barnes, A. Dereux, and T. W. Ebbesen, "Surface plasmon subwavelength optics," Nature, vol. 424, no. 6950, pp. 824830, 2006.

[2] S. Lal, S. Link, and N. J. Halas, "Nano-optics from sensing to wave guiding," Nature Photonics, vol. 1, no. 11, pp. 641-648, 2007.

[3] S. A. Maier, Plasmonics: fundamentals and applications, Springer, 1st edition, 2007.

[4] J. A. Schuller, E. S. Barnard, W. Cai, Y. C. Jun, J. S. White, and M. L. Brongersma, "Plasmonics for extreme light concentration and manipulation," Nature Materials, vol. 9, no. 3, pp. 193-204, 2010.

[5] J. M. Luther, P. K. Jain, T. Ewers, and A. P. Alivisatos, "Localized surface plasmon resonances arising from free carriers in doped quantum dots," Nature Materials, vol. 10, no. 5, pp. 361-366, 2011.

[6] Z. Fei, A. S. Rodin, G. O. Andreev et al., "Gate-tuning of graphene plasmons revealed by infrared nano-imaging," Nature, vol. 487, no. 7405, pp. 82-85, 2012.

[7] J. C. Weeber, J. R. Krenn, A. Dereux, B. Lamprecht, Y. Lacroute, and J. P. Goudonnet, "Near-field observation of surface plasmon polariton propagation on thin metal stripes," Physical Review B, vol. 64, no. 4, article 045411, 2001.

[8] P. Neutens, P. Van Dorpe, I. De Vlaminck, L. Lagae, and G. Borghs, "Electrical detection of confined gap plasmons in metal-insulator-metal waveguides," Nature Photonics, vol. 3, no. 5, pp. 283-286, 2009.

[9] L. Tang, S. E. Kocabas, S. Latif et al., "Nanometre-scale germanium photodetector enhanced by a near-infrared dipole antenna," Nature Photonics, vol. 2, no. 4, pp. 226229, 2008.

[10] A. Moreau, C. Ciracì, J. J. Mock et al., "Controlled-reflectance surfaces with film-coupled colloidal nanoantennas," Nature, vol. 492, no. 7427, pp. 86-89, 2012. 
[11] A. V. Kabashin, P. Evans, S. Pastkovsky et al., "Plasmonic nanorod metamaterials for biosensing," Nature Materials, vol. 8, no. 11, pp. 867-871, 2009.

[12] H. Gao, J.-C. Yang, J. Y. Lin et al., "Using the angle-dependent resonances of molded plasmonic crystals to improve the sensitivities of biosensors," Nano Letters, vol. 10, no. 7, pp. 25492554, 2010.

[13] Y. Shen, J. Zhou, T. Liu et al., "Plasmonic gold mushroom arrays with refractive index sensing figures of merit approaching the theoretical limit," Nature Communications, vol. 4, no. 1, p. 2381, 2013.

[14] H. A. Atwater and A. Polman, "Plasmonics for improved photovoltaic devices," Nature Materials, vol. 9, no. 3, pp. 205-213, 2010.

[15] D. Schurig, J. J. Mock, B. J. Justice et al., "Metamaterial electromagnetic cloak at microwave frequencies," Science, vol. 314, no. 5801, pp. 977-980, 2006.

[16] W. Cai, U. K. Chettiar, A. V. Kildishev, and V. M. Shalaev, "Optical cloaking with metamaterials," Nature Photonics, vol. 1, no. 4, pp. 224-227, 2007.

[17] X. Ni, Z. J. Wong, M. Mrejen, Y. Wang, and X. Zhang, "An ultrathin invisibility skin cloak for visible light," Science, vol. 349, no. 6254, pp. 1310-1314, 2015.

[18] J. C. Weeber, Y. Lacroute, and A. Dereux, "Optical near-field distributions of surface plasmon waveguide modes," Physical Review B, vol. 68, no. 11, article 115401, 2003.

[19] R. Zia and M. L. Brongersma, "Surface plasmon polariton analogue to Young's double-slit experiment," Nature Nanotechnology, vol. 2, no. 7, pp. 426-429, 2007.

[20] E. Verhagen, J. A. Dionne, L. (. K.). Kuipers, H. A. Atwater, and A. Polman, "Near-field visualization of strongly confined surface plasmon polaritons in metal-insulator-metal waveguides," Nano Letters, vol. 8, no. 9, pp. 2925-2929, 2008.

[21] T. Tanemura, K. C. Balram, D. S. Ly-Gagnon et al., "Multiplewavelength focusing of surface plasmons with a nonperiodic nanoslit coupler," Nano Letters, vol. 11, no. 7, pp. 2693-2698, 2011.

[22] J. Lin, J. Dellinger, P. Genevet, B. Cluzel, F. de Fornel, and F. Capasso, "Cosine-Gauss plasmon beam: a localized longrange nondiffracting surface wave," Physical Review Letters, vol. 109, no. 9, article 093904, 2012.

[23] L. Du, D. Y. Lei, G. Yuan et al., "Mapping plasmonic near-field profiles and interferences by surface-enhanced Raman scattering," Scientific Reports, vol. 3, no. 1, p. 3064, 2013.

[24] P. Genevet, D. Wintz, A. Ambrosio, A. She, R. Blanchard, and F. Capasso, "Controlled steering of Cherenkov surface plasmon wakes with a one-dimensional metamaterial," Nature Nanotechnology, vol. 10, no. 9, pp. 804-809, 2015.

[25] H. Ditlbacher, J. R. Krenn, N. Felidj et al., "Fluorescence imaging of surface plasmon fields," Applied Physics Letters, vol. 80, no. 3, pp. 404-406, 2002.

[26] H. Dong, S. R. du, X. Y. Zheng et al., "Lanthanide nanoparticles: from design toward bioimaging and therapy," Chemical Reviews, vol. 115, no. 19, pp. 10725-10815, 2015.

[27] B. Zhou, B. Shi, D. Jin, and X. Liu, "Controlling upconversion nanocrystals for emerging applications," Nature Nanotechnology, vol. 10, no. 11, pp. 924-936, 2015.

[28] A. Yin, Y. Zhang, L. Sun, and C. Yan, "Colloidal synthesis and blue based multicolor upconversion emissions of size and composition controlled monodisperse hexagonal $\mathrm{NaY}-$
$\mathrm{F}_{4}: \mathrm{Yb}, \mathrm{Tm}$ nanocrystals," Nanoscale, vol. 2, no. 6, pp. 953959, 2010.

[29] H. Zhang, D. Xu, Y. Huang, and X. Duan, "Highly spectral dependent enhancement of upconversion emission with sputtered gold island films," Chemical Communications, vol. 47, no. 3, pp. 979-981, 2011.

[30] Y. Liu, Y. Lu, X. Yang et al., "Amplified stimulated emission in upconversion nanoparticles for super-resolution nanoscopy," Nature, vol. 543, no. 7644, pp. 229-233, 2017.

[31] L. Salomon, G. Bassou, H. Aourag et al., "Local excitation of surface plasmon polaritons at discontinuities of a metal film: theoretical analysis and optical near-field measurements," Physical Review B, vol. 65, no. 12, article 125409, 2002.

[32] L. Zhang, A. Kubo, L. Wang, H. Petek, and T. Seideman, "Imaging of surface plasmon polariton fields excited at a nanometer-scale slit," Physical Review B, vol. 84, no. 24, article 245442, 2011.

[33] Y. Gong, A. G. Joly, D. Hu, P. Z. el-Khoury, and W. P. Hess, "Ultrafast imaging of surface plasmons propagating on a gold surface," Nano Letters, vol. 15, no. 5, pp. 34723478, 2015.

[34] I. P. Radko, S. I. Bozhevolnyi, G. Brucoli, L. Martín-Moreno, F. J. García-Vidal, and A. Boltasseva, "Efficiency of local surface plasmon polariton excitation on ridges," Physical Review $B$, vol. 78, no. 11, article 115115, 2018.

[35] J. Lin, J. P. B. Mueller, Q. Wang et al., "Polarization-controlled tunable directional coupling of surface plasmon polaritons," Science, vol. 340, no. 6130, pp. 331-334, 2013.

[36] R. Zia, M. D. Selker, and M. L. Brongersma, "Leaky and bound modes of surface plasmon waveguides," Physical Review B, vol. 71, no. 16, article 165431, 2005

[37] A. Lipson, S. G. Lipson, and H. Lipson, Optical physics, Cambridge University Press, 4th edition, 2011.

[38] K. V. Sreekanth, Y. Alapan, M. ElKabbash et al., "Extreme sensitivity biosensing platform based on hyperbolic metamaterials," Nature Materials, vol. 15, no. 6, pp. 621-627, 2016.

[39] A. J. Haes and R. P. Van Duyne, "A nanoscale optical blosensor: sensitivity and selectivity of an approach based on the localized surface plasmon resonance spectroscopy of triangular silver nanoparticles," Journal of the American Chemical Society, vol. 124, no. 35, pp. 10596-10604, 2002.

[40] K. M. Mayer and J. H. Hafner, "Localized surface plasmon resonance sensors," Chemical Reviews, vol. 111, no. 6, pp. 38283857, 2011.

[41] A. J. Haes and R. P. Van Duyne, "A unified view of propagating and localized surface plasmon resonance biosensors," Analytical and Bioanalytical Chemistry, vol. 379, no. 7-8, pp. 920930, 2004.

[42] G. Wu, R. H. Datar, K. M. Hansen, T. Thundat, R. J. Cote, and A. Majumdar, "Bioassay of prostate-specific antigen (PSA) using microcantilevers," Nature Biotechnology, vol. 19, no. 9, pp. 856-860, 2001.

[43] D. M. Rissin, C. W. Kan, T. G. Campbell et al., "Single-molecule enzyme-linked immunosorbent assay detects serum proteins at subfemtomolar concentrations," Nature Biotechnology, vol. 28, no. 6, pp. 595-599, 2010.

[44] L. Rodriguez-Lorenzo, R. de la Rica, R. A. Alvarez-Puebla, L. M. Liz-Marzan, and M. M. Stevens, "Plasmonic nanosensors with inverse sensitivity by means of enzyme-guided crystal growth," Nature Materials, vol. 11, no. 7, pp. 604-607, 2012. 
[45] R. de la Rica and M. M. Stevens, "Plasmonic ELISA for the ultrasensitive detection of disease biomarkers with the naked eye," Nature Nanotechnology, vol. 7, no. 12, pp. 821-824, 2012.

[46] G. Zheng, F. Patolsky, Y. Cui, W. U. Wang, and C. M. Lieber, "Multiplexed electrical detection of cancer markers with nanowire sensor arrays," Nature Biotechnology, vol. 23, no. 10, pp. 1294-1301, 2005.

[47] P. M. Kosaka, V. Pini, J. J. Ruz et al., "Detection of cancer biomarkers in serum using a hybrid mechanical and optoplasmonic nanosensor," Nature Nanotechnology, vol. 9, no. 12, pp. 1047-1053, 2014.

[48] J. M. Nam, C. S. Thaxton, and C. A. Mirkin, "Nanoparticlebased bio-bar codes for the ultrasensitive detection of proteins," Science, vol. 301, no. 5641, pp. 1884-1886, 2003.

[49] X. Liu, Q. Dai, L. Austin et al., “A one-step homogeneous immunoassay for cancer biomarker detection using gold nanoparticle probes coupled with dynamic light scattering," Journal of the American Chemical Society, vol. 130, no. 9, pp. 2780-2782, 2008.

[50] X. Wu, L. Xu, L. Liu et al., "Unexpected chirality of nanoparticle dimers and ultrasensitive chiroplasmonic bioanalysis," Journal of the American Chemical Society, vol. 135, no. 49, pp. 18629-18636, 2013. 